\title{
A non-standard statistical approach to the silo discharge
}

\author{
R. Arévalo ${ }^{1}$, A. Garcimartín ${ }^{1}$, and D. Maza ${ }^{2}$ \\ 1 Department of Physics and Applied Mathematics, Universidad de Navarra, \\ 31080 Pamplona, Spain \\ 2 Also at Physics Institute, Universidad de Navarra, 31080 Pamplona, Spain
}

\begin{abstract}
We present molecular dynamics simulations of the beginning of a silo discharge by gravity. The evolution of the velocity profile and the probability density functions for the displacements of the grains are obtained. These PDFs reveal non-gaussian statistics and superdiffusive behavior similar to that observed in some experiments. We propose an analytical expression for the PDFs and an explanation for its dynamical origin in connection with the ideas of the "spot" model and non-extensive thermodynamics.
\end{abstract}

\section{Introduction}

During the last few decades, interest has grown in the study of granular media in the framework of the physics of complex systems. Their complexity arises from the inelastic collisions through which their macroscopic constituents interact. The collisional regimes exhibited by them is therefore one of the topics that has been more deeply investigated. They can be broadly classified into two kinds: low and high density (respectively, fast and slow flows). The former has been amenable to be studied by means of the Boltzmann equation and further extensions -such as the Enskog equation [1]- and found to follow an hydrodynamic behavior [2]. But our understanding of the dense and slow flow, characterized by multiple collisions, the failure of molecular chaos hypothesis and the presence of correlations, is poorer, and a general theoretical framework fot its description is lacking. We focus our present work on this regime.

Dense flows are typically found in silos and Couette-type devices, both systems having been widely used to experimentally probe granular matter in this regime [3] [4]. The problem of the flow in a silo has been studied since long by engineers, both experimentally and theoretically [5]. As a result, the general properties of the flow are known and some models have been proposed to account for them. Classically, two different approaches are deemed successful for the explanation of the general features of the flow. One is the diffusive approach [6] [7], which is based in the movement of voids injected at the outlet of the silo. These voids diffuse upward, exchanging their positions with the grains which, in turn, move toward the orifice at the bottom. The other is the continuum approach [8] which, as implied by its name, is a continuum mechanics approximation in which the mean velocity field is the relevant variable.

New experimental results seem to limit the range of application of these models to the description of the overall and macroscopic behavior of the system when the flow is fully developed. In [9], a high-resolution particle-tracking experiment of the drainage of a quasi-2d silo is reported. The microscopics of grains movement during the discharge are characterized by super-diffusive behavior and non-gaussian statistics of the grains displacements at sufficiently short temporal resolution. The probability density functions (PDFs) are fat-tailed for both vertical and horizontal displacements. In addition, long correlations are found which are inconsistent with the physical image of the void model: a void passing near a grain would remove 
its contacts, thus limiting correlations to very short distances. The behavior depicted in the theoretical models is recovered as the discharge goes on. In that experiment, the PDFs of the displacements tend to a gaussian shape, and a super-diffusive to diffusive transition is observed in the movements of the grains once a displacement comparable to the particle diameter is attained. Measurements of the topological correlation function show that the correlations in the bulk of the silo decrease. This transition takes place approximately when the highest grain has fallen a distance twice its diameter.

Another important discrepancy between the discrete models and the observed dynamics is related to the experimentally determined "diffusion length". This length has been determined after the velocity profile measurements inside the silo [10] and in all the cases it is larger than the predicted for the models of voids diffusion.

In order to tackle this problem, M. Bazant [11] proposed an alternative model to interpret the results, assuming that the voids injected at the outlet of the silo do not preserve their shape. Instead, they spread through several grains which will move cooperatively. The resulting void spreading through a group of grains is called a "spot". Spots move upward inside the silo and the grains affected by the spot execute a small movement toward the base. The grains affected by the spot tend to move together, so this would explain the correlations observed and led to a diffusion length comparable to the one obtained experimentally. Nevertheless, the short lived superdiffusive regime is not well captured by this alternative model.

In the present work we report numerical simulations of a silo drainage. We analyze the evolution of the velocity profile at a temporal resolution larger that the experimental case. Two diffusive regimes can be clearly observed. The first one can be interpreted as showing "spots" for the particle displacement. In this regime we compile statistics on grains movements, showing that their movement is superdiffusive and their PDFs are non-gaussian, in agreement with experiments. Finally, we show that a statistical mechanics approach based in the existence of the "mass variable spots" can explain these results.

\section{Numerical simulations}

We perform soft-particle molecular dynamics simulations [12] of disks in two dimensions. The simulation starts with 5000 disks in a regular lattice having random velocities taken from a gaussian distribution. The disks fall under gravity through a conical hopper to a flat bottomed silo where they are deposited. We wait until all grains have fallen and most of their kinetic energy has been dissipated. We then open an outlet at the base allowing grains to fall, and we start to measure. In this protocol, the hopper is used to break the possible correlations arising from the initial configuration of the grains.

In our simulations both the hopper and the silo walls consist of the same type of grains that the medium. Their interactions are also the same and thus behave like rough walls that give rise to inelastic collisions. The flat silo is $50 \mathrm{~d}$ wide and reaches $100 \mathrm{~d}$ when filled, which guarantee that the flow is independent of both wall and fill effects. The height changes only slightly during the simulation.

The model for the forces consists of normal and transversal contacts as well as dissipative terms,

$$
\begin{array}{r}
F_{n}=k_{n} \xi^{3 / 2}-\gamma_{n} v_{i, j}^{n} \\
F_{t}=-\min \left(\mu\left|F_{n}\right|, \gamma_{s}\left|v_{i, j}^{s}\right|\right) \cdot \operatorname{sign}\left(v_{i, j}^{s}\right)
\end{array}
$$

where

$$
v_{i, j}^{s}=\dot{\mathbf{r}}_{i j} \cdot \mathbf{s}+\frac{1}{2} d\left(\omega_{i}+\omega_{j}\right)
$$

Equation (2.1) gives the force in the normal direction of the contact. The first term is a restoring force proportional to the superposition $\xi$ of the disks. The $3 / 2$ exponent arises from the Hertz theory of the contact. The second term is a dissipation proportional to the relative normal velocity of the interacting disks with damping coefficient $\gamma_{n}$. Eq. (2.2) is the sliding component 
of the damping force. It implements the Coulomb criterion with friction coefficient $\mu$. The damping in the transverse direction is proportional to the shear velocity given by Eq. (2.3) and a transverse damping coefficient $\gamma_{s}$. In this equation $\mathbf{s}$ is a unit vector tangential to the disks at the contact point and $\omega_{i}$ and $\omega_{j}$ are their angular velocities.

The values of the coefficients are, measured in reduced units, $k_{n}=10^{4} \mathrm{mg} / \mathrm{d}, \gamma_{n}=$ $100 m \sqrt{g / d}, \gamma_{s}=300 m \sqrt{g / d} \mu=0.5$. The integration time-step used is $1.25^{-4} \tau$ with, $\tau=\sqrt{d / g}$ and $m, d$ and $g$ stand respectively for the mass and diameter of the disks and the acceleration of gravity.

The equations of motion were integrated using the velocity-Verlet algorithm and a neighbor list was used to reduce the computational effort [18].

\section{Velocity profiles and probability density functions}

In this section we study the evolution of the vertical velocity field. Using two distinct diameters of the exit orifice, $3.8 d$ and $11 d$, we compute the velocity profile since the outlet is opened until the highest grain drops a distance twice its diameter. For each orifice diameter we perform 20 independent simulations and average them to obtain the final result. In Fig. 1 the evolution of the vertical velocity profile for the $11 d$ orifice is shown. It is evident that groups of grains
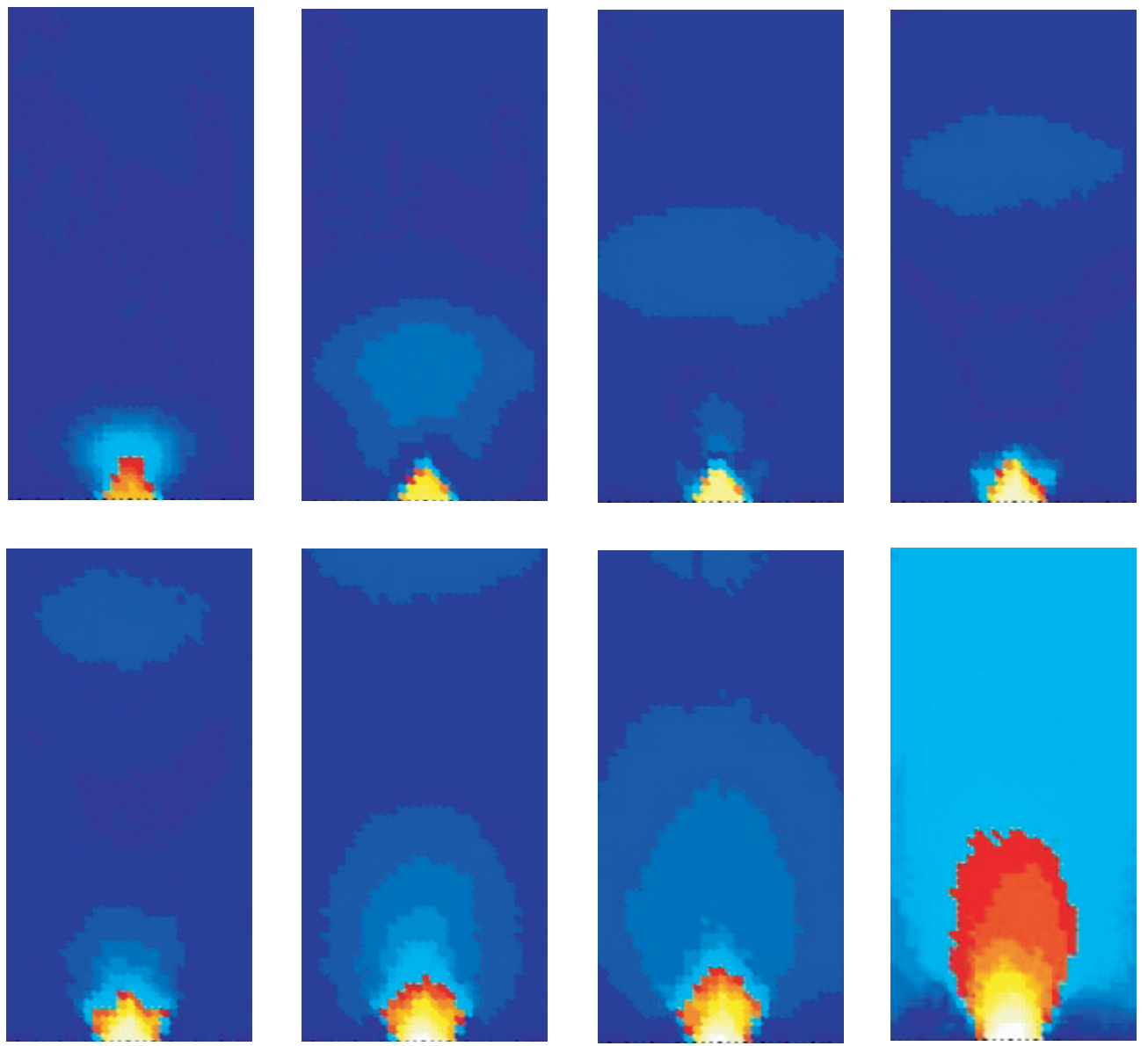

Fig. 1. Average vertical velocity profile inside the silo for an aperture $11 d$ in eight different stages of its evolution. Time increases from left to right and from top to bottom. In the last one - corresponding to the fully developed flow- a grain on top has fallen a distance equivalent to twice its diameter. Note the existence of a bounded region in the velocity profile that travel in the vertical direction. 
move downward together at the very beginning of the discharge, while structures resembling the image of spots introduced above can be seen moving upward. This scenario continues until eventually the characteristic stable flow profile is developed. The flow for the smaller exit orifice shares many common features. Nevertheless, there exists an important difference: the flow evolves more slowly, and spots can be seen even in the "stable" phase. This last observation is explained because the small used aperture induces an intermittent flow in the silo with tendency to jamming.

We also measured the displacements in vertical and horizontal directions of the individual grains. We chose to sample positions with a resolution of $0.01 d$, which is approximately the same used in the experiments [9]. We tracked all the grains that travel across a window with dimensions $15 d \times 15 d$ in the middle axis of the silo, the window being located in the upper half. To compute the PDFs of the vertical displacements we subtract the mean downward velocity of the flow. In Fig. 2 we show the normalized PDFs in semilogarithmic scale; displacements are rescaled in each direction by their standard deviation, which are of order $10^{-3} d$. We see that the PDFs are essentially the same for the vertical and horizontal displacements. Besides, they are clearly non-gaussian, with apparent differences both in the central region and in the tails -which are fat. Our curves (no analytic expression is given in previous experimental works) are very well described by the function

$$
P(\Delta y)=\frac{2 / \pi}{\left(1+(\Delta y)^{2}\right)^{2}}
$$

It is important to note that as the PDFs are normalized and the displacements rescaled by the standard deviation, there are no free parameters in the fits shown in the graphics.

(a)

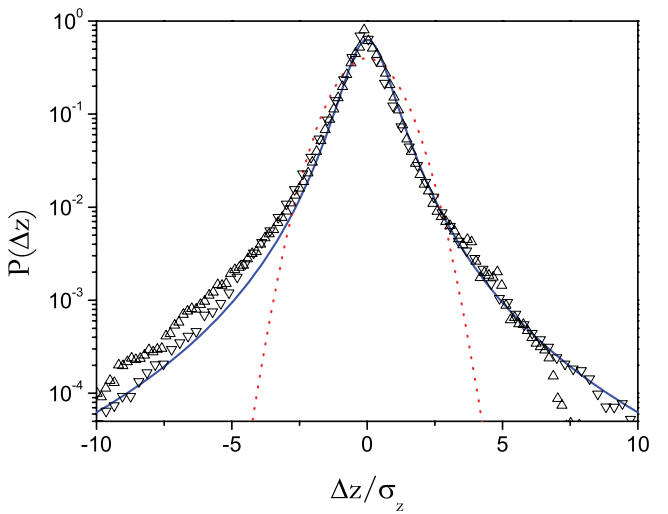

(b)

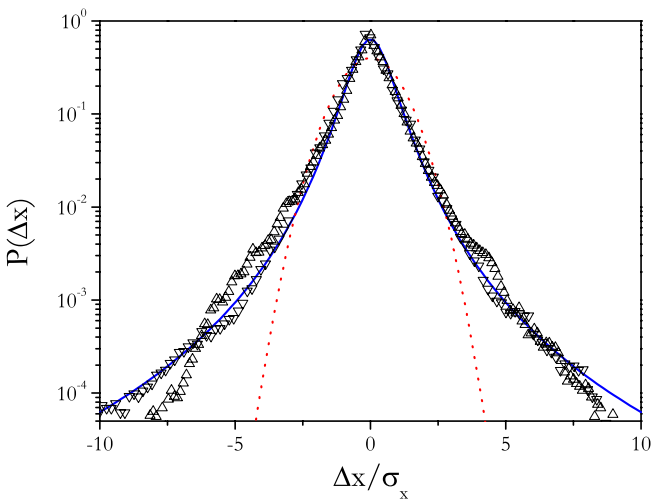

Fig. 2. Normalized PDFs for (a) horizontal and (b) vertical displacements. The symbols indicate the orifice aperture in the silo, up triangles for $11 d$ and down triangles for $3.8 d$. The dotted line is a gaussian and the solid line is Eq. (3.1).

In Fig. 3 we show the mean squared displacements in each direction. The slopes of the curves in a semilogarithmic scale are bigger than one, showing that the movement of the grains is super-diffusive. The mean squared displacements scale with $\left\langle x^{2}\right\rangle \propto t^{1.33}$ and $\left\langle z^{2}\right\rangle \propto t^{1.37}$ for the $3.8 d$ orifice, $\left\langle x^{2}\right\rangle \propto t^{1.5}$ and $\left\langle z^{2}\right\rangle \propto t^{1.51}$ for the $11 d$ orifice. These results are consistent with the values obtained experimentally.

\section{A non-standard statistical approach}

In this section we introduce an explanation for the origin of Eq. (3.1) in the basis of the existence of spots. To do so, we directly follow the ideas developed in [13] where the dynamics of a particle 
(a)

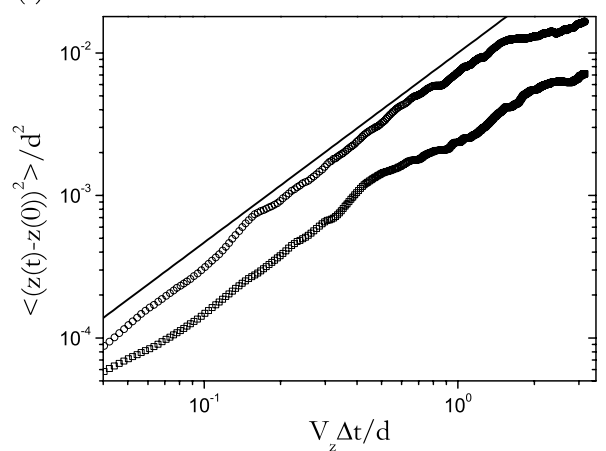

(b)

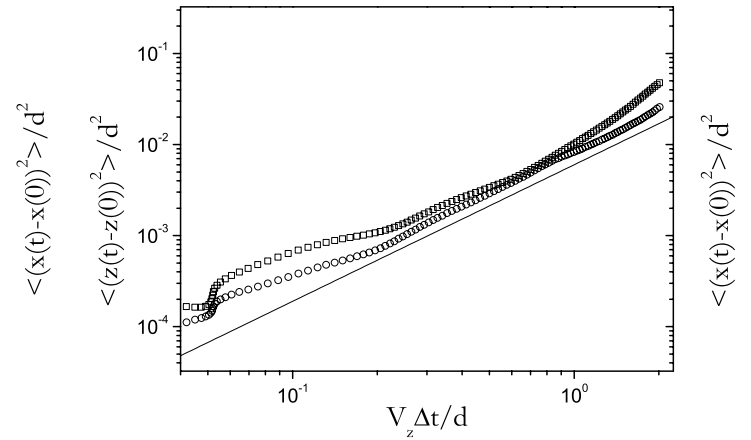

Fig. 3. Mean squared displacements in the horizontal and vertical directions in logarithmic scale. Figure (a) shows the results for an outlet size 3.8d, the line has a slope 1.33. Figure (b) shows the results for an outlet $11 d$ and the line has slope 1.5 (Note that at the early stages of the discharge the exponent it is a bit lower than 1.5. This fact is discussed in the text.

with a fluctuating mass is studied. Let us suppose that in our case the corresponding particle is a spot, whose mass fluctuates (as we can see from Fig. 1) as it moves inside the silo. The spot is a region of influence, so its boundary is not well determined or fixed and the number of grains affected by it may change as it evolves, thus making its mass fluctuate. Following [13] let us assume that the spot is a "Brownian particle having a fluctuating mass". Under this assumption we consider that (i) exists a distribution of masses $g(m)$ than can interact with the spot and (ii) the spot "mass" fluctuations are defined by a characteristic time $\tau$. For a given "spot mass" $m_{S}$ the spot velocity distribution converges to:

$$
f_{B}(u)=\sqrt{\frac{\beta m_{S}}{2 \pi}} e^{-\beta \frac{m_{S} u^{2}}{2}}
$$

The relaxation to that value will take place in a characteristic time scale $t_{R}$ dependent on the mass of the particle and the properties of the medium. If that time scale verifies $t_{R} \ll \tau$ then, the asymptotic distribution will be given by

$$
f_{B}(u)=\int d m g(m) \sqrt{\frac{\beta m_{S}}{2 \pi}} e^{-\beta \frac{m_{S} u^{2}}{2}}
$$

In order to proceed, we need a distribution of masses. As suggested in [13], a chi-squared distribution should be a good choice for this case:

$$
g(\beta)=\frac{1}{b \Gamma(c)}\left(\frac{\beta}{b}\right)^{c-1} e^{-\frac{\beta}{b}}
$$

The standard deviation of a variable is given by the average of the squared values it takes, if the average is zero (as in our case). The chi-squared is the distribution of a random variable defined as $\beta=\sum_{i=1}^{2 c} X_{i}^{2}$, where the $X_{i}$ are independent gaussian distributed variables with $\left\langle X_{i}\right\rangle=0$. The parameters $c$ and $b$ are related to the average and standard deviation of $\beta$.

We can now integrate Eq. (4.2) to obtain

$$
p(u)=\sqrt{\frac{b}{2 \pi}} \frac{\Gamma(c+1 / 2)}{\Gamma(c)} \frac{1}{\left(1+\frac{b}{2} u^{2}\right)^{c+1 / 2}}
$$

Imposing the same rescaling as in our data $\left\langle u^{2}\right\rangle=1$ implies $2 / b=1$ and if we choose $c=3 / 2$ Eq. (4.4) transforms into

$$
p(u)=\frac{\frac{2}{\pi}}{\left(1+u^{2}\right)^{2}}
$$

which is the same distribution function we found in our simulations. 
This result has been obtained for the spots, while the results given by the simulations Eq. (3.1) were obtained for individual grains. In order to join both descriptions we use the mathematical definition of a spot as given in [11]

$$
\Delta \mathbf{R}_{g}=-\omega\left(\mathbf{r}_{g}, \mathbf{r}_{s}\right) \Delta \mathbf{R}_{s}
$$

which means: when a spot moves $\Delta \mathbf{R}_{s}$ near $\mathbf{r}_{s}$ it produces a movement $\Delta \mathbf{R}_{g}$ in the grain which is in $\mathbf{r}_{g}$. The function $\omega$ is called the influence function and it spans over the radius of the spot. A simple estimation of its value based in volume conservation, assuming it is scalar and symmetric, yields $\omega=\Delta \phi / \phi^{2}$ where $\phi$ is the volume fraction. Let us assume a volume variation $\Delta \phi / \phi=1 \%$ and a density $\phi=0.8$, which is what we typically find in our simulations (monodisperse disks tend to be close packed [14]); then $\omega \simeq 10^{-3}$. If we introduce Eq. (4.6) in Eq. (4.5) we see that the influence function corresponds to the standard deviation in the movement of the individual grains. The estimated value of this function is the same obtained above for the standard deviation of the grains. Thus, starting with Eq. (4.5) for the spots and using Eq. (4.6) we recover exactly the PDF Eq. (3.1) for the individual grains.

Eq. (4.4) can be introduced in the formalism of nonextensive statistical mechanics [15]. Such function is commonly named as Tsallis distribution

$$
f(u)=\left(1+\frac{1}{2}(q-1) \beta^{\prime} u^{2}\right)^{\frac{1}{1-q}}
$$

with $c+\frac{1}{2}=\frac{1}{q-1}$ and $b=\beta^{\prime}(q-1)$. Our choice of $c$ in the Eq. (4.5) fixes the entropic index $q, c=3 / 2 \rightarrow q=3 / 2$. Indeed, the same results presented above can be obtained by directly applying the superstatistics procedure introduced by Beck [16] to the grain themselves.

It has been shown [17] how nonextensive statistical mechanics naturally arise when one considers the generalization of the diffusion equation

$$
\frac{\partial p(x, t)}{\partial t}=D \frac{\partial^{2} p(x, t)}{\partial x^{2}}
$$

Two such generalizations seem to be possible. One is to keep the equation linear and take fractional derivatives. This leads to solutions that are Lévy distributions characterized by diverging variance. The function we have found has finite variance, so these are not the functions we are interested in. The second way to generalize Eq. (4.8) is to make it nonlinear (NLDE)

$$
\frac{\partial p(x, t)}{\partial t}=D \frac{\partial^{2}[p(x, t)]^{\nu}}{\partial x^{2}}
$$

where $\nu$ is a real number. It is possible to solve this equation using the formalism of the nonextensive statistical mechanics. The solution is a Tsallis function with argument $x^{2}$ and the relation $q=2-\nu<3$ is verified. The second moment of this distribution is finite for every $q<5 / 3$, which is our case, and then the mean squared displacement scales with $\left\langle x^{2}\right\rangle \propto t^{2 /(3-q)}$. Such relation has been verified in same particular situations [19,20].

Thus for the value of $\alpha$ that we have found from the discharge process $\left\langle x^{2}\right\rangle \propto t^{4 / 3}=t^{1.33}$ it is possible to estimate the entropic parameter $q$ that correspond to the solution of the NLD Eq (4.9). This value is the same we obtained from the simulations with the smallest aperture size and is consistent with the slightly different value obtained for the bigger outlet. Nevertheless, for the last case, the scaling exponent seems to change as the time increase. This could be related to the fact that for outlet orifices large enough the relaxation time $t_{R}$ will have the same order of magnitude than the spot fluctuation time $\tau$.

\section{Discussion}

We have reported on numerical simulations of the beginning of a two-dimensional silo drainage process with two different aperture sizes. We check that the evolution of the velocity profile 
is consistent with the existence of spots or extended structures that give rise to cooperative motions and large correlations. The presence of these cage effects suggests that a reexamination of the microscopic foundation of the classical models for silo discharge is needed. As has been previously reported for the case of fully developed flows, we observe that the grains move superdiffusively for sufficiently lower scales and their displacements present non-gaussian statistics. Thus we introduce a appropriated function to fit the fluctuation distribution. The origin of this function can be explained in terms of spots if we consider them as a cluster of particles with variable mass. This procedure is based in a time scales separation: time scale for fluctuations must be greater than the relaxation time for a certain realization of the mass. As remarked above, the smaller the outlet orifice, the slower the evolution of spots is, so the separation of time scales is expected to be sharper for small orifices. This observation may explain why the function deduced fits better the results obtained for a small aperture.

It is interesting to note that this deduction is formally identical to the use of superstatistics arguments with individual grains. In this case we would consider the grains moving in a fluctuating medium which reaches local equilibrium faster than the typical time for the fluctuations of that medium. This picture rises doubts about the applicability of the concept of temperature to this system. Since the medium is fluctuating and a generalization of the Boltzmann factor (which is the foundation of superstatistics) is able to describe them, a temperature analog to the one defined in thermodynamics (proportional to the squared velocity of the grains) is not guaranteed to have the properties of a true temperature.

Finally, this picture allows us to interpret the value obtained for the entropic index. Above we noticed that $q=3 / 2 \rightarrow c=3 / 2$, and $2 c=3$ is the number of sums we take in the chi-squared distribution used. Note that we have obtained our results for the vertical and perpendicular directions separately, so we propose that 3 is, in average, the number of collisions a grain undergoes in each direction. We have measured the number of neighbors of individual grains while they fall inside the silo and found that this number is around 5.6 for both silos during the beginning of the discharge. The neighbors of a grain move around it and are not even always the same so, in average, we can assign three collisions to each direction.

This work has been supported by Project FI2005-03881 (MEC, Spanish Government), and PIUNA, University of Navarra. RA thanks Friends of the University of Navarre for a fellowship.

\section{References}

1. N.V. Brilliantov, T. Pöschel, Kinetic Theory of Granular Gases (Oxford University Press, Oxford, 2004)

2. J.J. Brey, J.W. Dufty, C.S. Kim, A. Santos, Phys. Rev. E 58, 4638 (1998)

3. I. Zuriguel, A. Garcimartín, D. Maza, L.A. Pugnaloni, J.M. Pastor, Phys. Rev. E 71, 051303 (2005)

4. B. Utter, R.P. Behringer, Phys. Rev. E 69, 031308 (2004)

5. R.M. Nedderman, Statics and kinematics of granular materials (Nova Science, 1991)

6. J. Litwiniszyn, Bull. Acad. Pol. Sci. 11, 593 (1963)

7. J. Mullins, Powder Technol. 9, 29 (1974)

8. R.M. Nedderman, U. Tüzün, Powder Technol. 22, 243 (1979)

9. J. Choi, A. Kudrolli, R.R. Rosales, M.Z. Bazant, Phys. Rev. Lett. 92, 174301 (2004)

10. J. Choi, A. Kudrolli, M.Z. Bazant, J. Phys. Cond. Matt. 17, S2533-S2548 (2005)

11. M.Z. Bazant, Mech. Mat. 38, 717 (2006)

12. J. Schäfer, S. Dippel, D.E. Wolf, J. Phys. I (France), 6, 5 (1996)

13. M. Ausloos, R. Lambiotte, Phys. Rev. E 73, 011105 (2006)

14. R. Arévalo, D. Maza, L.A. Pugnaloni, Phys. Rev. E 74, 021303 (2006)

15. C. Tsallis, J. Stat. Phys. 52, 479 (1998)

16. C. Beck, Phys. Rev. Lett. 87180601 (2001)

17. C. Tsallis, A.M.C. de Souza, R. Maynard, Lévy Flights and Related Topics in Physics, edited by M. Schlesinger, G.M. Zaslavsky, U. Frisch (Springer, Berlin Heidelberg, New York, 1995), p. 269

18. D.C. Rappaport, The art of molecular dynamics simulation (Cambridge University Press, 2004)

19. H. Viridissima, I. Upadhyaya, J.-P. Rieu, J. Glaizer, Y. Sawada, Physica A 293, 549 (2001)

20. K. Daniels, C. Beck, E. Bodenschatz, Physica D 193, 208 (2004) 
